\title{
Improving engagement with healthcare in hepatitis C: a randomised controlled trial of a peer support intervention
}

\author{
Helen R. Stagg ${ }^{1,2^{*}}$, Julian Surey ${ }^{1}$, Marie Francis ${ }^{1}$, Jennifer MacLellan ${ }^{1}$, Graham R. Foster ${ }^{3}$, André Charlett ${ }^{4}$ and
} Ibrahim Abubakar ${ }^{1 *}$

\begin{abstract}
Background: Peer support can enable patient engagement with healthcare services, particularly for marginalised populations. In this randomised controlled trial, the efficacy of a peer support intervention at promoting successful engagement with clinical services for chronic hepatitis $C$ was assessed.

Methods: In London, UK, potential participants were approached through outreach services for problematic drug use and homelessness. Individuals positive for hepatitis $\mathrm{C}$ virus (HCV) after confirmatory testing were randomised using an online service to the intervention (peer support) or standard of care. The primary outcome of interest was successful engagement with clinical hepatitis services. The study was non-blinded. Absolute differences were calculated using a generalised linear model and the results compared to logistic regression.

Results: Three hundred sixty-four individuals consented to participate. One hundred one had chronic hepatitis C and were randomised, 63 to receive the intervention (peer support). A successful outcome was achieved by 23 individuals in this arm (36.5\%) and seven (18.4\%) receiving the standard of care, giving an absolute increase of $18.1 \%$ (95\% confidence interval $1.0-35.2 \%, p$ value $=0.04$ ). This was mirrored in the logistic regression (odds ratio $2.55(0.97-6.70), p=0.06)$. No serious adverse events were reported.
\end{abstract}

Conclusions: Peer support can improve the engagement of patients with chronic HCV with healthcare services. Trial registration: ISRCTN24707359. Registered 19th October 2012.

Keywords: Hepatitis C, Peer support, Peer advocacy, Peer worker

\section{Background}

In England, around 160,000 individuals were estimated to be chronically infected with hepatitis $\mathrm{C}$ virus $(\mathrm{HCV})$ in 2017, a large proportion of whom are from underserved and marginalised groups [1]. The UK has committed to eliminating HCV by 2025 [2]; thus, it is critical that all population groups are able to access effective diagnostic and treatment services.

Underserved and vulnerable groups are defined by factors that render engagement with normal healthcare

* Correspondence: helen.stagg@ed.ac.uk; i.abubakar@ucl.ac.uk

IInstitute for Global Health, University College London, 4th floor, Mortimer Market Centre, off Capper Street, London WC1E 6JB, UK

${ }^{2}$ Usher Institute of Population Health Sciences and Informatics, The University of Edinburgh, MacKenzie House, 30 West Richmond Street, Edinburgh EH8 9DX, UK

Full list of author information is available at the end of the article services and pathways problematic, which often results in them being labelled as 'hard-to-reach' by such systems. They include homeless persons, people who inject drugs and ex-prisoners. These groups can be vulnerable to a range of infectious diseases due to living conditions, exposure to injecting drug use, alcoholism, generally poor physical and mental health and sociostructural factors that criminalise, isolate and stigmatise. Exposure to, and prevalence of, hepatitis B virus (HBV) and $\mathrm{HCV}$ among marginalised groups in the $\mathrm{UK}$ is known to be high [3-5]. Whilst testing for $\mathrm{HCV}$ and HBV is not uncommonly offered by UK outreach services, this is not sufficient to promote effective engagement through the full diagnostic and treatment process.

(c) The Author(s). 2019 Open Access This article is distributed under the terms of the Creative Commons Attribution 4.0 International License (http://creativecommons.org/licenses/by/4.0/), which permits unrestricted use, distribution, and 
The treatment landscape for chronic HCV has been revolutionised in the past decade, with the licencing of a series of directly acting antivirals (DAAs) for HCV [6]. Although expensive, these drugs have radically improved the patient treatment experience, shortened the duration of therapy, reduced the likelihood of adverse events and have better (pan-genotype) treatment success rates. Such regimens offer hope to all chronically infected individuals, but most especially those who may struggle to access specialist services and adhere to treatment. Monthly treatment targets of chronic HCV patients have been set for each Operational Delivery Network $(\mathrm{ODN})$ in England; as hospitals work through their lists of patients awaiting DAAs, greater efforts will be needed to reach the individuals less likely to engage [7]. This includes those currently injecting drugs, a key population if elimination of $\mathrm{HCV}$ is to be achieved. Underserved and vulnerable groups as a whole will act as a reservoir of infection if their healthcare needs are not addressed.

Peer support is a mechanism to enable active engagement with healthcare among marginalised groups. Peers have personal experience of a specific illness or lifestyle that enables them to support others experiencing similar challenges [8]. Peer support has been used worldwide, particularly in mental health, to remove barriers to accessing services, facilitate patient wellbeing and promote positive clinical outcomes [8]. In chronic hepatitis $\mathrm{C}$, peers have been documented as playing a wide range of roles [9-11]; helping individuals to engage with their treatment is a particularly critical area $[12,13]$. Qualitative and observational studies have highlighted peer support models as valuable in the care of chronically infected HCV patients in Australia, Georgia, the UK and the USA [12-25], but quantitative trial data is lacking. Peer support has been deemed one of ten priorities to improve access to HCV treatment for people who inject drugs in low- and middle-income countries [26].

Given the promising observational data and qualitative evidence, and the absence of quantitative trial data on the value of peer support for promoting successful engagement with clinical services for chronic $\mathrm{HCV}$, we conducted a randomised controlled trial (RCT) aimed to evaluate the efficacy of an individual-level peer support intervention.

\section{Methods}

\section{Study design}

This was a randomised non-blinded controlled trial in London, UK, to assess the efficacy of a community-controlled, individual-level, peer support intervention to promote engagement with healthcare services in individuals chronically infected with $\mathrm{HCV}$.

\section{Participants}

Potential participants were approached at outreach services for problematic drug use and homelessness for point-of-care $\mathrm{HCV}, \mathrm{HBV}$, and $\mathrm{HIV}$ testing. Inclusion criteria were being marginalised by normal healthcare services (evidenced by engagement with outreach services as a client), over the age of 16 years, and willing and able to provide written informed consent. Potential participants were excluded if they were already on treatment for $\mathrm{HCV}$ or $\mathrm{HBV}$. Additionally, individuals known by outreach services to be positive for $\mathrm{HCV}$ and/or $\mathrm{HBV}$ who were not on treatment ('known positives') were approached.

\section{Testing procedures}

All consenting participants were asked to complete a baseline questionnaire (Additional file 1) by the study Research Nurses, which collected self-reported demographic, medical and contact information. They were offered point-of-care testing for $\mathrm{HCV}$ (OraQuick anti-HCV), HBV (nal von Minden HBsAg) and HIV (Alere third generation). Participants could choose to have less than the full complement of tests.

Participants found to be point-of-care test positive for $\mathrm{HCV}$ or $\mathrm{HBV}$ were informed at the time of screening and had venous and/or dried blood spot samples of blood taken for confirmatory testing by Public Health England (PHE), which provides a national reference service for $\mathrm{HCV}$ and $\mathrm{HBV}$ testing. Confirmatory antibody testing for chronic infection was undertaken for $\mathrm{HCV}$ and antigen/antibody testing for $\mathrm{HBV}$; if positive, this was followed by polymerase chain reaction (PCR) testing for genetic material and genotyping. Individuals positive for HIV at the point-of-care testing stage were informed and the results provided to their primary care practitioner, if permission was given.

Anyone testing positive for $\mathrm{HCV}$ or $\mathrm{HBV}$ at this confirmatory stage was fully enrolled into the trial and randomised to the intervention or standard care arms.

\section{Randomisation and masking}

Randomisation was undertaken centrally by the research nurses using the web-based Sealed Envelope system. Eligible enrolled participants were randomised 1:2 standard of care to intervention arm. Additional details are presented in Additional file 2, including the sample size calculation.

\section{Standard of care}

Enrolled participants not randomised to the intervention arm were referred to one of four hospitals (The Royal London/Barts Health, King's College London, Royal Free, University College). Their test results-and notification of their study participation-were sent to their primary care practitioner, if permission was given. Individuals were allowed to choose which hospital to be 
referred to, regardless of their study arm. There was no further intervention by the trial team. The specific site of referral for both arms of the study was chosen based on participant preference and geographical location prior to randomisation results being known. Referral sites did not explicitly know the randomisation status of the patients.

\section{Intervention}

In our community-controlled model of peer support, participants in the intervention arm were individually assigned to a peer advocate from the London-based homeless charity and advocacy organisation Groundswell. The details of the development of this intervention are presented in Additional file 2.

\section{Outcomes and follow-up}

The primary outcome of interest was successful achievement of an appropriate clinical endpoint, defined as engagement with clinical hepatitis services i.e. three engagements within 6 months of the first booked clinical appointment. An engagement could be a review with a doctor or nurse, FibroScan or ultrasound scan, or a blood test. This number was set after consultation with clinical colleagues to demonstrate a real willingness of the participant to engage.

The secondary trial outcomes for enrolled participants were (a) successfully reaching a sustained virological response (SVR; an undetectable viral load for 6 months after the end of treatment) against HCV and (b) successful engagement, reaching a full clinical diagnosis, or commencing treatment for HBV.

Outcome data collection and the process for withdrawals are described in more detail in Additional file 2.

\section{Statistical analysis}

Data collection and cleaning are described in detail in Additional file 2.

Following descriptive analyses of the baseline population, absolute differences in the proportion of participants successfully achieving three engagements in the intervention and control arms were calculated as an intention-to-treat analysis using a generalised linear model assuming a binomial distribution and an identity link function. The results of this model were compared to that of a logistic regression model. Additional details about the building of these models are presented in Additional file 2.

After examining the distribution of individuals who withdrew or became lost to follow-up (LFU), a post hoc per protocol sensitivity analysis was planned where people in the intervention arm who withdrew or became LFU before they had a peer advocate assigned were re-categorised into the standard of care arm.
Descriptive analyses were undertaken for the secondary outcomes of interest, due to the small numbers of relevant patients.

\section{Results}

\section{Baseline study population}

Between 15 August 2013 and 10 June 2015, 364 individuals across a total of 27 outreach services in London consented to point-of-care testing (Fig. 1). Recruitment was stopped when we reached our sample size, allowing for delays in obtaining confirmatory PCR results. Follow-up was completed on 29 April 2016. The baseline characteristics of the individuals who consented are presented in Table 1.

Consenting participants were largely male (278/364, $76.4 \%$ ), with a median age of 43 years (interquartile range $35-48)$, born in the UK $(276 / 364,75.8 \%)$ and of White other or White central/eastern European ethnicity (258/364, 76.4\%). High levels of key social risk factors associated with being at risk for HCV infection were displayed, including 82/364 (22.5\%) of participants being current injecting users of illicit drugs. The majority of participants were active smokers (321/364, 88.2\%). Around three quarters (272/364) had previously been tested for $\mathrm{HCV}$ or $\mathrm{HBV}$ and over a third previously diagnosed with HBV, HCV or another form of liver disease (136/364). Fifty-four individuals (14.8\%) were recruited as 'known positives' from services.

Of these 364 individuals, 136 had a positive pointof-care test for $\mathrm{HCV}$, three for HBV and three for HIV. Chronic HBV infection was confirmed in one individual by PCR testing, who was enrolled and placed in the intervention arm. One hundred one individuals were enrolled with a chronic HCV infection by PCR. This population was similar to the overall study population, but a greater proportion of individuals were from a White other ethnic background (70/101, 69.3\%), current users of illicit drugs (79/101, 78.2\% versus $213 / 364$, $58.5 \%$ ) or displayers of other social risk factors; had been previously tested or diagnosed; or were 'known positives' (54/101, 53.5\% versus 54/364, $14.8 \%$ ).

Sixty-three individuals were randomised to receive the intervention and 38 to the standard of care arm (Fig. 1). The distribution of baseline characteristics was not found to be appreciably different between randomisation arms among the 101 individuals positive for a chronic $\mathrm{HCV}$ infection who were enrolled (Table 1).

\section{Withdrawals and losses to follow-up}

Three individuals withdrew post-randomisation during the study, one from the standard of care and two from the intervention arm (Fig. 1). The characteristics of those who withdrew were not substantially different from those who did not (Additional file 3). Fifty-nine individuals were 




LFU, three of whom were in the intervention arm and whom became LFU before a peer advocate was assigned (Fig. 1). Their characteristics were not substantially different from the overall enrolled population, apart from when it came to a history of imprisonment: more had been imprisoned in the last 5 years (Additional file 3).

\section{Successful engagement with clinical services}

$50.5 \%$ of the HCV-positive individuals (51/101) engaged with services at least once within 6 months of their first booked clinical appointment (median one, maximum six, interquartile range $0-3) .29 .7 \%(30 / 101)$ achieved a successful outcome, i.e. engaged with services three times or more. Of these 30 individuals, seven were in the standard of care arm $(7 / 38,18.4 \%)$ and $23(23 / 63$, $36.5 \%)$ were in the intervention arm. Their baseline characteristics are documented in Table 2.

Outcomes by peer advocate were investigated as part of the process to determine the potential need to adjust for clustering during the regression analysis. Of the 63 individuals in the intervention arm, five were not assigned a peer advocate, two due to withdrawing and three due to becoming LFU before assignment could occur. Of the remaining 58 individuals, peer advocate $\mathrm{A}$ was assigned to the greatest number (33/58, 56.9\%; Additional file 4). Although there was variation in terms of the proportions of individuals cared for by each peer advocate that reached a successful outcome, the confidence intervals (CIs) around each percentage overlapped with the overall percentage.

Univariable regression models were thus built without adjustment for clustering by peer advocate to examine the relationship between the intervention and successfully engaging. In the model of absolute differences, patients in the intervention arm were found to have an $18.1 \%(95 \%$ CI $1.0 \%-35.2 \%, p$ value $=0.04)$ increased likelihood of a successful treatment outcome versus those in the standard of care arm. In the model of relative differences, the odds of reaching a successful treatment outcome were 2.55 times higher ( $95 \%$ CI $0.97-6.70, p=0.06$ ) among individuals in the intervention arm versus those in the standard of care arm.

Although imbalances by randomisation arm had not been detected in baseline characteristics, bivariable models adjusting for potential confounders were created. Point estimates differed (Additional file 5), but the CIs for each model overlapped with that of the univariable model for both absolute and relative measures. A Lowess plot of age versus outcomes revealed a nonlinear relationship and thus both age and age squared were included in the relevant model. Imprisonment and sex had particularly large impacts in terms of drawing the CIs towards the null. Smoking and HIV status were collinear with the outcome and thus could not be assessed. 
Table 1 Baseline characteristics of consenting participants

\begin{tabular}{|c|c|c|c|c|c|c|c|}
\hline \multirow[t]{2}{*}{ Characteristic } & \multicolumn{2}{|c|}{ Overall } & \multicolumn{2}{|c|}{ Enrolled^ } & \multicolumn{3}{|c|}{ Intervention^ } \\
\hline & No. & Col. $\%$ & No. & Col. $\%$ & No. & Col. \% & $p$ \\
\hline Overall & 364 & 100.0 & 101 & 100.0 & 63 & 100.0 & \\
\hline Sex & & & & & & & 0.45 \\
\hline Male & 278 & 76.4 & 81 & 80.2 & 52 & 82.5 & \\
\hline Female & 86 & 23.6 & 20 & 19.8 & 11 & 17.5 & \\
\hline Missing & 0 & 0.0 & 0 & 0.0 & 0 & 0.0 & \\
\hline Age categorised (years) & & & & & & & 0.58 \\
\hline $16-25$ & 22 & 6.0 & 1 & 1.0 & 1 & 1.6 & \\
\hline $26-35$ & 79 & 21.7 & 16 & 15.8 & 10 & 15.9 & \\
\hline $36-45$ & 130 & 35.7 & 42 & 41.6 & 23 & 36.5 & \\
\hline $46-55$ & 105 & 28.8 & 35 & 34.7 & 25 & 39.7 & \\
\hline $56-65$ & 27 & 7.4 & 6 & 5.9 & 3 & 4.8 & \\
\hline $66-75$ & 1 & 0.3 & 1 & 1.0 & 1 & 1.6 & \\
\hline Missing & 0 & 0.0 & 0 & 0.0 & 0 & 0.0 & \\
\hline Ethnicity & & & & & & & 0.59 \\
\hline White other & 173 & 47.5 & 70 & 69.3 & 42 & 66.7 & \\
\hline White central/eastern European & 85 & 23.4 & 9 & 8.9 & 6 & 9.5 & \\
\hline Indian subcontinent & 5 & 1.4 & 1 & 1.0 & 0 & 0.0 & \\
\hline Black & 72 & 19.8 & 12 & 11.9 & 9 & 14.3 & \\
\hline Mixed/other & 27 & 7.4 & 8 & 7.9 & 6 & 9.5 & \\
\hline Missing & 2 & 0.5 & 1 & 1.0 & 0 & 0.0 & \\
\hline UK born & & & & & & & $>0.99$ \\
\hline No & 88 & 24.2 & 23 & 22.8 & 14 & 22.2 & \\
\hline Yes & 276 & 75.8 & 78 & 77.2 & 49 & 77.8 & \\
\hline Missing & 0 & 0.0 & 0 & 0.0 & 0 & 0.0 & \\
\hline Use of illicit drugs & & & & & & & 0.56 \\
\hline Absent & 69 & 19.0 & 1 & 1.0 & 1 & 1.6 & \\
\hline Present, but unknown what/when & 1 & 0.3 & 21 & 20.8 & 14 & 22.2 & \\
\hline Present, previous & 81 & 22.3 & 0 & 0.0 & 0 & 0.0 & \\
\hline Present, current non-injecting & 131 & 36.0 & 47 & 46.5 & 26 & 41.3 & \\
\hline Present, current injecting & 82 & 22.5 & 32 & 31.7 & 22 & 34.9 & \\
\hline Missing & 0 & 0.0 & 0 & 0.0 & 0 & 0.0 & \\
\hline Homelessness & & & & & & & 0.92 \\
\hline Absent & 51 & 14.0 & 15 & 14.9 & 10 & 15.9 & \\
\hline Present, previous & 112 & 30.8 & 51 & 50.5 & 32 & 50.8 & \\
\hline Present, current & 200 & 54.9 & 35 & 34.7 & 21 & 33.3 & \\
\hline Missing & 1 & 0.3 & 0 & 0.0 & 0 & 0.0 & \\
\hline Imprisonment & & & & & & & 0.34 \\
\hline Absent & 128 & 35.2 & 16 & 15.8 & 10 & 15.9 & \\
\hline Present, $>5$ years ago & 103 & 28.3 & 47 & 46.5 & 33 & 52.4 & \\
\hline Present, $\leq 5$ years ago & 132 & 36.3 & 37 & 36.6 & 20 & 31.7 & \\
\hline Missing & 1 & 0.3 & 1 & 1.0 & 0 & 0.0 & \\
\hline Alcohol-related concerns & & & & & & & 0.68 \\
\hline
\end{tabular}

Absent, not sure or missing 
Table 1 Baseline characteristics of consenting participants (Continued)

\begin{tabular}{|c|c|c|c|c|c|c|c|}
\hline \multirow[t]{2}{*}{ Characteristic } & \multicolumn{2}{|c|}{ Overall } & \multicolumn{2}{|c|}{ Enrolled^ } & \multicolumn{3}{|c|}{ Intervention^ } \\
\hline & No. & Col. \% & No. & Col. \% & No. & Col. \% & $p$ \\
\hline Present & 216 & 59.3 & 56 & 55.4 & 36 & 57.1 & \\
\hline Smoking & & & & & & & $-^{*}$ \\
\hline Current & 321 & 88.2 & 100 & 99.0 & 62 & 98.4 & \\
\hline Ex-smoker & 14 & 3.8 & 0 & 0.0 & 0 & 0.0 & \\
\hline Missing & 29 & 8.0 & 1 & 1.0 & 1 & 1.6 & \\
\hline HIV status & & & & & & & $>0.99$ \\
\hline Negative or not sure & 360 & 98.9 & 99 & 98.0 & 62 & 98.4 & \\
\hline Positive & 4 & 1.1 & 2 & 2.0 & 1 & 1.6 & \\
\hline HBV vaccination status & & & & & & & 0.81 \\
\hline Not vaccinated or not sure & 137 & 37.6 & 24 & 23.8 & 16 & 25.4 & \\
\hline One or more doses & 227 & 62.4 & 77 & 76.2 & 47 & 74.6 & \\
\hline Previous testing & & & & & & & 0.29 \\
\hline Not tested or not sure & 92 & 25.3 & 4 & 4.0 & 4 & 6.3 & \\
\hline Yes, for HBV or HCV & 272 & 74.7 & 97 & 96.0 & 59 & 93.7 & \\
\hline Previous diagnosis & & & & & & & 0.79 \\
\hline Not diagnosed or not sure & 228 & 62.6 & 19 & 18.8 & 11 & 17.5 & \\
\hline Yes, for HBV, HCV or liver disease & 136 & 37.4 & 82 & 81.2 & 52 & 82.5 & \\
\hline 'Known positive' at the time of recruitment & & & & & & & 0.54 \\
\hline No & 310 & 85.2 & 47 & 46.5 & 31 & 49.2 & \\
\hline Yes & 54 & 14.8 & 54 & 53.5 & 32 & 50.8 & \\
\hline \multicolumn{8}{|l|}{ HCV point-of-care test result } \\
\hline Negative & 224 & 61.5 & & & & & \\
\hline Positive & 136 & 37.4 & & & & & \\
\hline Not done (refused) & 3 & 0.8 & & & & & \\
\hline Borderline & 1 & 0.3 & & & & & \\
\hline \multicolumn{8}{|l|}{ HBV point-of-care test result } \\
\hline Negative & 306 & 84.1 & & & & & \\
\hline Positive & 3 & 0.8 & & & & & \\
\hline Not done (refused) & 46 & 12.6 & & & & & \\
\hline Test failed & 9 & 2.5 & & & & & \\
\hline \multicolumn{8}{|l|}{ HIV point-of-care test result } \\
\hline Negative & 334 & 91.8 & & & & & \\
\hline Positive & 3 & 0.8 & & & & & \\
\hline Not done (refused) & 27 & 7.4 & & & & & \\
\hline
\end{tabular}

$\wedge$ Excluding one individual positive for HBV but not HCV at confirmatory testing. *Everyone in both randomisation arms with information available was a current smoker. Col column, HBV hepatitis B virus, HCV hepatitis $C$ virus, $p$ Fisher's exact test $p$ value (missing values excluded) comparing individuals in the intervention and standard of care arms

There was no evidence of effect modification by any of the predefined variables. Tests for interaction failed for ethnicity, being UK born, use of illicit drugs and previous testing due to small numbers in particular strata.

\section{Sensitivity analyses}

Ad hoc per protocol analyses were undertaken for both the absolute and relative models to determine the impact of initial non-assignation of a peer advocate to individuals in the intervention arm due to withdrawal or LFU. Assigning affected individuals to the standard of care arm, the absolute difference in the percentage likelihood of achieving a successful treatment outcome increased to $23.4 \%$ (95\% CI $6.6-$ $40.1 \%, p=0.01)$ and the relative difference to 3.38 (95\% CI 1.29-8.88, $p=0.01$ ). 
Table 2 Outcomes by study arm and baseline characteristics

\begin{tabular}{lll}
\hline Characteristic & \multicolumn{2}{l}{$\geq 3$ engagements } \\
\cline { 2 - 3 } & No. & Row \% \\
\hline Overall & 30 & 297
\end{tabular}

Randomisation

Standard of care

Intervention

Sex

Male

Female

Missing

Age categorised (years)

16-25

26-35

36-45

46-55

56-65

66-75

Missing

Ethnicity

White other

White central/eastern European

Indian subcontinent

Black

Mixed/other

Missing

UK born

No

Yes

Missing

Use of illicit drugs

Absent

Present, but unknown what/when

Present, previous

Present, current non-injecting

Present, current injecting

Missing

Homelessness

Absent

Present, previous

Present, current

Missing

Imprisonment

Absent

Present, $>5$ years ago

Present, $\leq 5$ years ago

7

Table 2 Outcomes by study arm and baseline characteristics (Continued)

\begin{tabular}{|c|c|c|}
\hline \multirow[t]{2}{*}{ Characteristic } & \multicolumn{2}{|c|}{$\geq 3$ engagements } \\
\hline & No. & Row \% \\
\hline Missing & 0 & 0.0 \\
\hline \multicolumn{3}{|l|}{ Alcohol-related concerns } \\
\hline Absent, not sure or missing & 14 & 31.1 \\
\hline Present & 16 & 28.6 \\
\hline \multicolumn{3}{|l|}{ Smoking } \\
\hline Current & 30 & 30.0 \\
\hline Ex-smoker & 0 & - \\
\hline Missing & 0 & 0.0 \\
\hline \multicolumn{3}{|l|}{ HIV status } \\
\hline Negative or not sure & 30 & 30.3 \\
\hline Positive & 0 & 0.0 \\
\hline \multicolumn{3}{|l|}{ HBV vaccination status } \\
\hline Not vaccinated or not sure & 8 & 33.3 \\
\hline One or more doses & 22 & 28.6 \\
\hline \multicolumn{3}{|l|}{ Previous testing } \\
\hline Not tested or not sure & 1 & 25.0 \\
\hline Yes, for HBV or HCV & 29 & 29.9 \\
\hline \multicolumn{3}{|l|}{ Previous diagnosis } \\
\hline Not diagnosed or not sure & 5 & 26.3 \\
\hline Yes, for HBV, HCV or liver disease & 25 & 30.5 \\
\hline \multicolumn{3}{|c|}{ 'Known positive' at the time of recruitment } \\
\hline No & 11 & 23.4 \\
\hline Yes & 19 & 35.2 \\
\hline
\end{tabular}

Excluding one individual positive for $\mathrm{HBV}$ but not $\mathrm{HCV}$ at confirmatory testing. $H B V$ hepatitis $B$ virus, $H C V$ hepatitis $C$ virus

\section{Secondary outcomes}

Among individuals with a chronic HCV infection, none achieved a SVR during the study period. The individual diagnosed with chronic HBV assigned to the intervention arm did not achieve a successful outcome of three or more engagements with healthcare services within six months; they became LFU eight months after their first appointment (with no subsequent appointments).

\section{Adverse events}

No serious adverse events were documented within the study.

\section{Discussion}

In a RCT of an individual-level, community-controlled, peer support intervention to promote successful engagement with clinical services for individuals diagnosed with chronic $\mathrm{HCV}$, we demonstrate that providing patients with a peer advocate increased their absolute likelihood of successfully engaging with healthcare systems by 
18.1\%. In a logistic regression model, this translated into 2.55 times the odds of a successful outcome, albeit with a CI that minimally crossed the null.

Importantly, an ad hoc per protocol sensitivity analysis revealed the impact of not staying within the study for long enough to be assigned a peer advocate. Within this population, arrest and imprisonment were key reasons for becoming LFU. When individuals in the intervention arm who were never assigned a peer advocate were analysed as though they were in the standard of care arm both effect sizes and statistical measures of association were strengthened.

We believe this to be the first RCT to evaluate the efficacy of a peer support intervention at improving engagement with healthcare services for chronic HCV. The closest previous study found in the literature was a randomised pilot trial in Belgium of an educational intervention that included a peer component and use of FibroScan screening [27]. This study was small (52 participants) and did not detect an enduring change in willingness to be treated in the intervention versus control arms. A number of descriptive observational studies have documented the role of different peer models in HCV testing and treatment [17, 19, 22-25], but provided no formal proof of the causal effects of the implemented interventions.

Some limitations of the study should be considered. The study was not blinded, meaning that participants were aware of the trial arm that they were assigned to, as were study staff. This could have altered participant behaviour and the way that the outcome was documented during data collection, biasing the effect estimate away from the null, although data collection was undertaken using standardised forms and processes across all patients. Data were only collected from the hospitals that the patients initially chose to attend. It is possible that patients in the standard of care arm in particular changed hospital during their engagement with services, biasing the effect estimate away from the null. Given that such patients would have likely had to re-start their diagnostic process from scratch, however, this can still be seen as a failure within the clinical system. Originally, only three peer advocates were planned to be involved in the intervention arm, but eight were actually utilised. As we found no evidence of clustering by peer, this is unlikely to have reduced study power. Our peer advocates were all male, but we found no evidence for effect modification by participant sex within our analysis. No patients achieved a SVR during the study, partly due to delays placing individuals on treatment as a result of changes in the regimens that were available within national healthcare services during the RCT. During the trial, treatment was additionally limited to those with higher FibroScan scores. This prevented us from analysing the impact of peer support on treatment success rates.

Since 2016, ODNs have been responsible for delivering $\mathrm{HCV}$ treatment in England, with monthly treatment targets that should promote access to DAAs for marginalised individuals. This expansion of treatment comes with its own challenges regarding ensuring patient engagement with the diagnostic and treatment pathway. This study provides high-quality evidence that peer advocates may provide an efficacious way of promoting this, in line with global calls for the use of peer support models in chronic HCV [26].

\section{Conclusions}

In conclusion, our findings indicate that peer support can improve the engagement of patients with chronic $\mathrm{HCV}$ with healthcare services. The cost-effectiveness of such interventions in the relevant populations should be assessed before they are implemented in different healthcare settings.

\section{Additional files}

Additional file 1: Study questionnaire. Copy of questionnaire used during study. (DOCX $771 \mathrm{~kb}$ )

Additional file 2: Detailed methodology. Detailed methods for the trial. (DOCX $27 \mathrm{~kb}$ )

Additional file 3: Baseline characteristics of individuals who withdrew or were lost to follow up. Tabulated baseline information for study participants who were lost to follow-up or who withdrew. (DOCX $22 \mathrm{~kb}$ )

Additional file 4: Outcomes by peer advocate. Tabulated study outcomes, stratified by peer advocate. (DOCX $18 \mathrm{~kb}$ )

Additional file 5: Regression analysis of effectiveness of peer support intervention in Hepatitis C Virus-positive individuals, adjusted for potential confounding. Tabulated additional analyses. (DOCX 19 kb)

\section{Abbreviations}

Cl: Confidence interval; DAAs: Directly acting antivirals; GP: General practitioner (primary care provider); HBV: Hepatitis B virus; HCV: Hepatitis C virus; HHPA: Homeless Health Peer Advocacy; LFU: Lost to follow-up; ODN: Operational Delivery Network; PCR: Polymerase chain reaction; PHE: Public Health England; RCT: Randomised controlled trial; SVR: Sustained virological response

\section{Acknowledgements}

The authors thank Groundswell for their support of this work (particularly Kate Bowgett; patient and public involvement), the Trial Steering Committee (Professor Paul Hunter, University of East Anglia; Dr. Stefan Lozewicz, North Middlesex/Barts and the London Hospitals; Emma Ward/Rachel Halford, Hepatitis C Trust- patient and public involvement) and the Data Monitoring Committee (Dr. Mark Kroese, PHG Foundation; Dr. Cono Ariti, London School of Hygiene and Tropical Medicine; Dr. David Adeboyeku, Central Middlesex Hospital).

\section{Funding}

This report is independent research funded by the National Institute for Health Research Policy Research Programme ('Effectiveness of testing for treatment of hard-to-reach groups for latent tuberculosis, hepatitis B virus and hepatitis C virus in England: The HALT Study', 015/0306). The views expressed in this publication are those of the author(s) and not necessarily those of the NHS, the National Institute for Health Research or the Department of Health and Social Care. The study funders and sponsor had no role 
in the design of the study; the collection, analysis, and interpretation of the data; in the writing of the paper; and in the decision to submit it for publication.

\section{Availability of data and materials}

The datasets used and/or analysed during the current study are not publicly available as consent for data sharing was not obtained at enrolment.

\section{Authors' contributions}

IA conceived of and designed the work with input from AC, GRF and HRS. JS, MF and JM collected the data. AC, HRS and IA analysed the data. All authors interpreted the data. HRS, AC and IA drafted the paper. All authors revised the work critically for important intellectual content. All authors agree to be accountable for all aspects of the work and approved the final version to be published.

\section{Ethics approval and consent to participate}

The study was reviewed and approved by the National Research Ethics Service Committee London, Brent (13/LO/0077), UK. All participants gave written informed consent to be part of the study.

\section{Consent for publication}

Not applicable.

\section{Competing interests}

HRS reports funding from the Department of Health during the course of the study. JS, JM and MF report funding from the Department of Health and the National Institute for Health Research Clinical Research Network, UK, during the conduct of the study. GF reports personal fees from AbbVie, Gilead and Merck during the conduct of the study. The other authors declare that they have no competing interests.

\section{Publisher's Note}

Springer Nature remains neutral with regard to jurisdictional claims in published maps and institutional affiliations.

\section{Author details}

${ }^{1}$ Institute for Global Health, University College London, 4th floor, Mortimer Market Centre, off Capper Street, London WC1E 6JB, UK. ${ }^{2}$ Usher Institute of Population Health Sciences and Informatics, The University of Edinburgh, MacKenzie House, 30 West Richmond Street, Edinburgh EH8 9DX, UK. ${ }^{3}$ Barts Liver Centre, Blizard Institute, Queen Mary University of London, London E1 2AT, UK. ${ }^{4}$ Statistics, Modelling and Economics Department, Public Health England, 61 Colindale Avenue, London NW9 5EQ, UK.

Received: 23 October 2018 Accepted: 6 March 2019

Published online: 01 April 2019

\section{References}

1. Public Health England. Hepatitis C in England: 2017 report. 2017. https:// www.gov.uk/government/uploads/system/uploads/attachment_data/file/ 599738/hepatitis_c_in_england_2017_report.pdf. Accessed 23 Oct 2018.

2. NHS England. NHS England sets out plans to be first in the world to eliminate Hepatitis C. 2018. https://www.england.nhs.uk/2018/01/ hepatitis-c-2/. Accessed 23 Oct 2018.

3. Neale J. Homelessness, drug use and hepatitis C: a complex problem explored within the context of social exclusion. Int J Drug Policy. 2008;19(6):429-35.

4. Peate I. Prisoner health: HIV infection and other blood-borne viral infections. Br J Nurs. 2011;20(10):605-6 608, 610.

5. Public Health England, Health Protection Scotland, Public Health Wales, Public Health Agency Northern Ireland. Shooting up: infections among people who inject drugs in the UK 2012. 2013. http://webarchive. nationalarchives.gov.uk/20140629102627/http://hpa.org.uk/Publications/ InfectiousDiseases/BloodBornelnfections/ShootingUp/ 1311ShootinguplnfectionsamongIDUS/. Accessed 23 Oct 2018.

6. World Health Organization. Guidelines for the screening care and treatment of persons with chronic hepatitis C infection. 2016. http://apps.who.int/iris/ bitstream/10665/205035/1/9789241549615 eng.pdf?ua=1. Accessed 23 Oct 2018.
7. Foster $G$, Huskinson P. 25,000 hepatitis $C$ patients receive new treatments. 2018. https://www.england.nhs.uk/blog/25000-hepatitis-c-patients-receivenew-treatments/. Accessed 23 Oct 2018.

8. MacLellan J, Surey J, Abubakar I, Stagg HR. Peer support workers in health: a qualitative metasynthesis of their experiences. PLoS One. 2015; 10(10):e0141122.

9. Kresina TF, Sylvestre D, Seeff L, Litwin AH, Hoffman K, Lubran R, et al. Hepatitis infection in the treatment of opioid dependence and abuse. Subst Abuse. 2008;1:15-61.

10. Grebely J, Tyndall MW. Management of HCV and HIV infections among people who inject drugs. Curr Opin HIV AIDS. 2011;6(6):501-7.

11. Henderson C, Madden A, Kelsall J. 'Beyond the willing \& the waiting' - the role of peer-based approaches in hepatitis $C$ diagnosis \& treatment. Int J Drug Policy. 2017;50:111-5

12. Mehta SH, Genberg BL, Astemborski J, Kavasery R, Kirk GD, Vlahov D, et al. Limited uptake of hepatitis $C$ treatment among injection drug users. J Community Health. 2008;33(3):126-33.

13. Robaeys G, Grebely J, Mauss S, Bruggmann P, Moussalli J, De Gottardi A, et al. Recommendations for the management of hepatitis $C$ virus infection among people who inject drugs. Clin Infect Dis. 2013;57(Suppl 2):S129-37.

14. Norman J, Walsh NM, Mugavin J, Stoove MA, Kelsall J, Austin K, et al. The acceptability and feasibility of peer worker support role in community based HCV treatment for injecting drug users. Harm Reduct J. 2008;5:8.

15. Treloar C, Jackson C, Gray R, Newland J, Wilson H, Saunders V, et al. Care and treatment of hepatitis $C$ among aboriginal people in New South Wales, Australia: implications for the implementation of new treatments. Ethn Health. 2016;21(1):39-57

16. Manos MM, Ho CK, Murphy RC, Shvachko VA. Physical, social, and psychological consequences of treatment for hepatitis C: a communitybased evaluation of patient-reported outcomes. Patient. 2013;6(1):23-34.

17. Sylvestre DL, Zweben JE. Integrating HCV services for drug users: a model to improve engagement and outcomes. Int J Drug Policy. 2007;18(5):406-10.

18. Batchelder AW, Cockerham-Colas L, Peyser D, Reynoso SP, Soloway I, Litwin $\mathrm{AH}$. Perceived benefits of the hepatitis $\mathrm{C}$ peer educators: a qualitative investigation. Harm Reduct J. 2017;14(1):67.

19. Grebely J, Knight E, Genoway KA, Viljoen M, Khara M, Elliott D, et al. Optimizing assessment and treatment for hepatitis $C$ virus infection in illicit drug users: a novel model incorporating multidisciplinary care and peer support. Eur J Gastroenterol Hepatol. 2010;22(3):270-7.

20. Bonnington $\mathrm{O}$, Harris $\mathrm{M}$. Tensions in relation: how peer support is experienced and received in a hepatitis $C$ treatment intervention. Int J Drug Policy. 2017;47:221-9.

21. Treloar C, Rance J, Bath N, Everingham H, Micallef M, Day C, et al. Evaluation of two community-controlled peer support services for assessment and treatment of hepatitis C virus infection in opioid substitution treatment clinics: the ETHOS study, Australia. Int J Drug Policy. 2015;26(10):992-8.

22. Roose RJ, Cockerham-Colas L, Soloway I, Batchelder A, Litwin AH. Reducing barriers to hepatitis $C$ treatment among drug users: an integrated hepatitis $C$ peer education and support program. J Health Care Poor Underserved. 2014;25(2):652-62.

23. Keats J, Micallef M, Grebely J, Hazelwood S, Everingham H, Shrestha N, et al. Assessment and delivery of treatment for hepatitis $C$ virus infection in an opioid substitution treatment clinic with integrated peer-based support in Newcastle, Australia. Int J Drug Policy. 2015;26(10):999-1006.

24. Kikvidze T, Luhmann N, Avril E, Butsashvili M, Labartkava K, Etienne A, et al. Harm reduction-based and peer-supported hepatitis $C$ treatment for people who inject drugs in Georgia. Int J Drug Policy. 2018;52:16-9.

25. Grebely J, Genoway K, Khara M, Duncan F, Viljoen M, Elliott D, et al. Treatment uptake and outcomes among current and former injection drug users receiving directly observed therapy within a multidisciplinary group model for the treatment of hepatitis C virus infection. Int J Drug Policy. 2007;18(5):437-43.

26. Ford N, Wiktor S, Kaplan K, Andrieux-Meyer I, Hill A, Radhakrishnan P, et al. Ten priorities for expanding access to HCV treatment for people who inject drugs in low- and middle-income countries. Int J Drug Policy. 2015;26(11):1088-93.

27. Arain A, De Sousa J, Corten K, Verrando R, Thijs H, Mathei C, et al. Pilot study: combining formal and peer education with FibroScan to increase HCV screening and treatment in persons who use drugs. J Subst Abus Treat. 2016;67:44-9. 